\title{
Symmetric Parsec-Scale Radio Jets in NGC 4261
}

Dayton L. Jones

Jet Propulsion Laboratory, Caltech, Pasadena, CA, USA

Ann E. Wehrle

Infrared Processing and Analysis Center, JPL, Caltech, Pasadena, CA, USA

\begin{abstract}
VLBA observations of NGC 4261 (3C 270) reveal highly symmetric radio structures at both 1.6 and $8.4 \mathrm{GHz}$. There is little evidence for free-free absorption in the inner few pc, despite the fact that HST imaging shows this galaxy to contain a nearly edge-on disk of gas and dust in its nucleus. However, at our highest resolution we find a narrow gap in emission just east of the radio core which we interpret as evidence for a small (sub-parsec) nearly edge-on accretion disk which is obscuring the base of the counterjet. The position angle of the pc-scale radio axis agrees with the position angle of the VLA-scale jets, which differs from the apparent rotation axis of the nuclear disk seen by HST.
\end{abstract}

\section{Introduction}

NGC 4261 hosts a classical FR I radio source (3C 270) whose symmetric kpcscale jets lie along a position angle of $88 \pm 1^{\circ}$ (Birkinshaw \& Davis 1985). HST observations have revealed that the optical nucleus of this galaxy is surrounded by a disk of gas and dust with a projected rotation axis that differs from the radio jet axis by several degrees (Ferrarese, Ford, \& Jaffe 1996). Our initial motivation for observing NGC 4261 with VLBI was to see if the parsec-scale radio structure was aligned with the kpc-scale jets or with the rotation axis of the HST disk.

\section{Observations and Results}

We observed NGC 4261 with the VLBA in April 1995 at both 1.6 and $8.4 \mathrm{GHz}$. At both frequencies we find basically symmetric two-sided jets aligned with the $\mathrm{kpc}$-scale jets imaged by the VLA. This implies that the plane of the innermost accretion disk (at the base of the radio jets) differs from the plane of the 100 pc-scale HST disk.

Analysis if the VLBA images shows that most of the central $10 \mathrm{pc}$ are not significantly affected by free-free absorption, a surprising finding since the largerscale HST disk appears to be within $20-30^{\circ}$ of edge-on. The implied electron density over the inner $10 \mathrm{pc}$ is less than $\sim 10^{3} \mathrm{~cm}^{-3}$ assuming a temperature of $\sim 10^{4} \mathrm{~K}$.

Our highest resolution images (see figure 1) show a very narrow gap in emission just east of the brightest peak. The peak corresponds to the core of the radio source based on spectral index distribution, and the gap appears on the side of the receding jet based on the brightness of the VLA jets and the orientation of the HST disk. This is the expected signature of absorption by a thin inner accretion disk seen nearly edge-on. If the inclination of this inner disk is close to that of the larger-scale HST disk it becomes optically thin to 8.4 $\mathrm{GHz}$ radiation at a deprojected radius of $0.8 \mathrm{pc}$. The thickness of the disk must be less than $0.1 \mathrm{pc}$. The much lower angular resolution at $1.6 \mathrm{GHz}$ prevents this 
feature from being detectable. Proposed higher frequency VLBA observations should allow us to determine the radial electron density distribution in the disk (e.g., Walker et al., these Proceedings, p. 133).

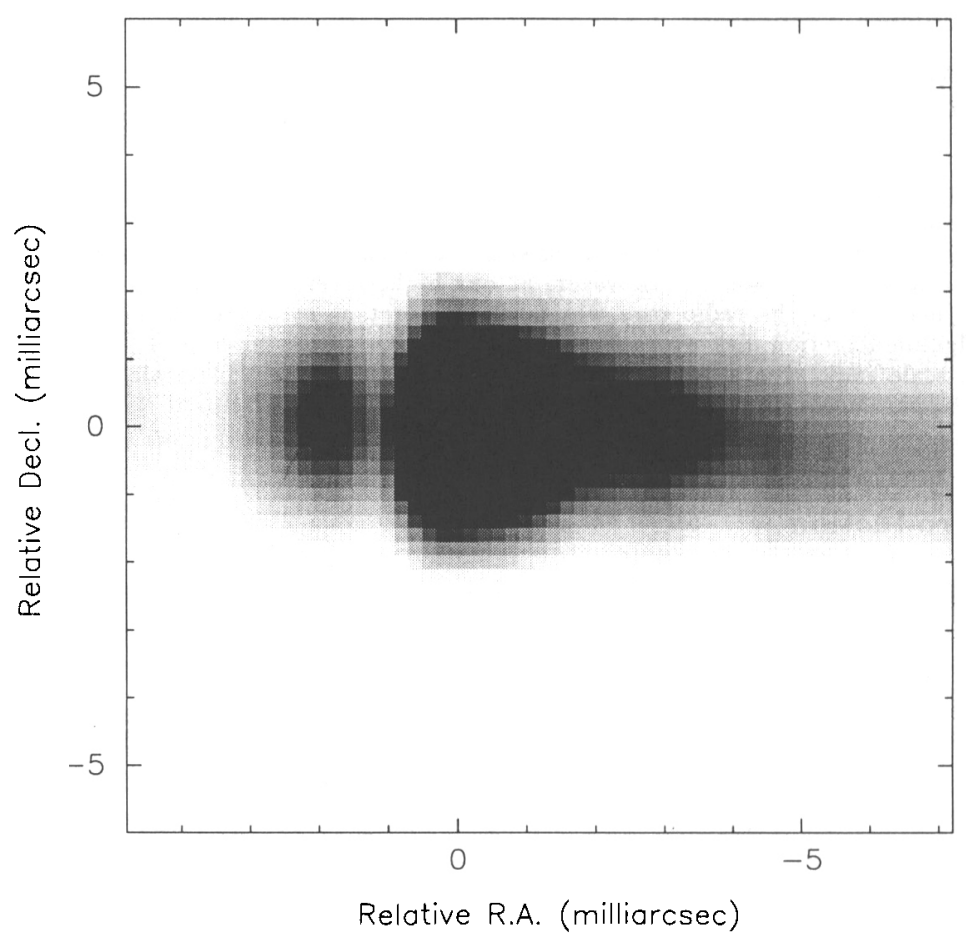

Figure 1. VLBA image of NGC 4261 at $8.4 \mathrm{GHz}$ showing the "gap" in emission just east of the brightest peak (core; origin of coordinates). Contour plots show a drop in brightness just east of the core which is consistent with a step function convolved with the restoring beam.

It appears that there is a small dense inner accretion disk centered on the radio core (the base of the jets; $<1 \mathrm{pc}$ ), a low density region filling most of the inner several pc of the nucleus (within which the radio jet brightness decreases rapidly in both directions, suggesting rapid expansion), and a surrounding cool higher density region of which the HST disk is part (and within which the rate of decrease in jet brightness is nearly halted; $>10 \mathrm{pc}$ ).

Acknowledgments. This research was carried out at the Jet Propulsion Laboratory, California Institute of Technology, under contract with the National Aeronautics and Space Administration. The National Radio Astronomy Observatory is a facility of the National Science Foundation, operated under a cooperative agreement by Associated Universities, Inc.

\section{References}

Birkinshaw, M., \& Davis, R. L. 1985. ApJ, 291, 32-44.

Ferrarese, L., Ford, H. C., \& Jaffe, W. 1996. ApJ, 470, 444-459. 